
Preprints des Instituts für Mathematik der Universität Potsdam 

Preprints des Instituts für Mathematik der Universität Potsdam 5 (2016) 1

\section{A Hilbert Boundary Value Problem for Generalised Cauchy-Riemann Equations}


Bibliografische Information der Deutschen Nationalbibliothek

Die Deutsche Nationalbibliothek verzeichnet diese Publikation in der Deutschen Nationalbibliografie; detaillierte bibliografische Daten sind im Internet über http://dnb.dnb.de abrufbar.

\section{Universitätsverlag Potsdam 2016}

http://verlag.ub.uni-potsdam.de/

Am Neuen Palais 10, 14469 Potsdam

Tel.: +49 (0)331 9772533 / Fax: 2292

E-Mail: verlag@uni-potsdam.de

Die Schriftenreihe Preprints des Instituts für Mathematik der Universität Potsdam wird herausgegeben vom Institut für Mathematik der Universität Potsdam.

ISSN (online) 2193-6943

Kontakt:

Institut für Mathematik

Karl-Liebknecht-Straße 24/25

14476 Potsdam

Tel.: +49 (0)331977 1499

WWW: http://www.math.uni-potsdam.de

Titelabbildungen:

1. Karla Fritze | Institutsgebäude auf dem Campus Neues Palais

2. Nicolas Curien, Wendelin Werner | Random hyperbolic triangulation

Published at: http://arxiv.org/abs/1105.5089

Das Manuskript ist urheberrechtlich geschützt.

Online veröffentlicht auf dem Publikationsserver der Universität Potsdam

URL https://publishup.uni-potsdam.de/opus4-ubp/frontdoor/index/index/docId/8610

URN urn:nbn:de:kobv:517-opus4-86109

http://nbn-resolving.de/urn:nbn:de:kobv:517-opus4-86109 


\title{
A HILBERT BOUNDARY VALUE PROBLEM FOR GENERALISED CAUCHY-RIEMANN EQUATIONS
}

\author{
AMMAR ALSAEDY AND NIKOLAI TARKHANOV
}

\begin{abstract}
We elaborate a boundary Fourier method for studying an analogue of the Hilbert problem for analytic functions within the framework of generalised Cauchy-Riemann equations. The boundary value problem need not satisfy the Shapiro-Lopatinskij condition and so it fails to be Fredholm in Sobolev spaces. We show a solvability condition of the Hilbert problem, which looks like those for ill-posed problems, and construct an explicit formula for approximate solutions.
\end{abstract}

\section{Contents}

Introduction

Part 1. Preliminary results

1. Generalised Cauchy-Riemann equations 3

2. The Hilbert boundary value problem 5

3. A Hilbert problem for the Fueter equations 6

4. Green formula 8

5. Hardy spaces 9

Part 2. Boundary Fourier method 11

6. Operator-theoretic foundations 11

7. The Cauchy problem 13

8. The Fischer-Riesz equations 14

9. Regularisation of solutions 17

10. Solvability of elliptic boundary value problems 19

References 20

\section{INTRODUCTION}

In [AT13] we develop the method of Fischer-Riesz equations for the study of boundary value problems for overdetermined elliptic systems of general type. For an abstract framework of this method we refer the reader to [Tar95, Ch. 11]. A

Date: December 22, 2015.

2010 Mathematics Subject Classification. Primary 35F45; Secondary 35J56, 47N20.

Key words and phrases. Dirac operator, Clifford algebra, Riemann-Hilbert problem, Fredholm operators.

The first author gratefully acknowledges the financial support of the Deutscher Akademischer Austauschdienst. 
leasing designation of the method is perhaps the boundary Fourier method. The central point of this method is the construction of a system of solutions to the formal adjoint system which is complete in the complementary space of Cauchy data. An explicit construction is given within the framework of approximation by solutions of underdetermined elliptic systems. However, a system obtained in this way is complete if and only if the boundary value problem in question has at most one solution. This is usually the case for the Cauchy problem with data on a nonempty boundary piece but need not hold even for Fredholm boundary value problems.

The purpose of the present paper is to elaborate the boundary Fourier method in the case where the problem fails to have a unique solution. To show the efficiency of the method we restrict our discussion to a very particular boundary value problem for generalised Cauchy-Riemann equations, which is an analogue of the Hilbert problem, see [Ste91] and elsewhere.

The classical Hilbert problem for holomorphic functions consists in the following, see [Gak77, § 27]. Given any continuous real-valued function $u_{0}$ on the boundary of a bounded domain $\mathcal{X} \subset \mathbb{C}$, find a holomorphic function $u=u_{1}+\imath u_{2}$ in $\mathcal{X}$ whose real and imaginary parts have limit values on the contour $\partial \mathcal{X}$ satisfying $b_{1} u_{1}+b_{2} u_{2}=u_{0}$. The coefficients $b_{1}, b_{2}$ of this linear combination are assumed to be given real-valued functions on $\partial \mathcal{X}$.

The comprehensive theory of the classical Riemann and Hilbert problems owes much to the works of Muskhelishvili and his school, see [Mus46], [Vek62], etc. Nowadays there have been studied several nonlinear versions of the classical problems, see [Shn72], [Weg92].

The concept of generalised Cauchy-Riemann equations is usually related to harmonic analysis in the Euclidean space. To the best of our knowledge, an analogue of the classical Cauchy-Riemann equations in the three-dimensional space was first shown in [MT31]. The paper [Fue36] then initiated a systematic study of functions of one quaternionic variable and thus their counterparts within the more general framework of hypercomplex numbers, see [Fre56], [SW68], [BDS82], [GM90], [OT08] and references given there. Boundary value problems for generalised Cauchy-Riemann systems in the space and their lower order perturbations were investigated in [Ste93a, Ste93b], [SV95], etc.

The generalised Cauchy-Riemann equations constitute a special subclass of the so-called Dirac operators, by which are meant matrix factorisations of the LaplaceBeltrami operator on a Riemannian manifold. The determined (i.e., given by square matrices) Dirac operators are first-order elliptic partial differential operators, and so there are rigid relations between the dimension $n$ of the underlying manifold and the number $k$ of unknown real-valued functions, see [Bal02]. In particular, the number $k$ is always even, and so the Hilbert problem for a Dirac operator $A$ consists in finding a solution $u$ to $A u=f$ in a relatively compact domain $\mathcal{X}$ satisfying $B u=u_{0}$ at the boundary of $\mathcal{X}$, where $B$ is a $(k / 2 \times k)$-matrix of realvalued functions of maximal rank $k / 2$.

Boundary value problems for Dirac operators are studied in [BW92], still, this book focuses on spectral problems. The general theory of [Vol65] does not apply to the Hilbert problem, for this latter need not satisfy the Shapiro-Lopatinskij condition. On applying the boundary Fourier method we derive explicit solvability conditions and a formula for an approximate solution of the Hilbert problem for 
generalised Cauchy-Riemann equations in the space. A criterion for the Fredholm property of the Hilbert problem is established in [Ste91]. In [Ste93a] this criterion is used to investigate the equation on existence and nonexistence of Fredholm boundary value problems. The paper [Ste93b] studies the adjoint problem to establish conditions which imply the uniqueness of the solution and the solvability for arbitrary data. Even in this case our approach has the advantage of providing an approximate solution.

\section{Part 1. Preliminary results}

\section{Generalised Cauchy-Riemann equations}

Consider a system of first order partial differential equations with constant coefficients of the form

$$
A_{1} \partial_{1} u+\ldots+A_{n} \partial_{n} u=0
$$

in $\mathbb{R}^{n}$, where $A_{1}, \ldots, A_{n}$ are $(k \times k)$-matrices of complex numbers, $\partial_{j}$ the partial derivative in the $j$ th coordinate $x^{j}$, for $j=1, \ldots, n$, and $u$ is an unknown function with values in $\mathbb{C}^{k}$. We will write $A$ for the partial differential operator on the left-hand side of (1.1).

After [SW68], the system (1.1) is called a generalised Cauchy-Riemann system if each solution $u$ to (1.1) has only harmonic components $u^{j}$.

In particular, if there is a $(k \times k)$-matrix $B$ of first-order partial differential operators on $\mathbb{R}^{n}$ satisfying $B A=E_{k} \Delta$, then $A$ is a generalised Cauchy-Riemann system.

Lemma 1.1. Every generalised Cauchy-Riemann system of the form (1.1) is elliptic, i.e., its symbol

$$
\sigma(A)(\xi):=\sum_{j=1}^{n} A_{j}\left(\imath \xi_{j}\right)
$$

is invertible for all $\xi \in \mathbb{R}^{n} \backslash\{0\}$.

Proof. See Theorem 1.1 of [Ste91]. If the lemma were wrong, then there would be $\xi \in \mathbb{R}^{n} \backslash\{0\}$ and $v \in \mathbb{C}^{k} \backslash\{0\}$, such that $\left(A_{1} \xi_{1}+\ldots+A_{n} \xi_{n}\right) v=0$. Hence it follows by straightforward calculations that $u=e^{\langle\xi, x\rangle} v$ satisfies $A u=0$ while $\Delta u=|\xi|^{2} u$ is different from zero. Therefore, (1.1) can not be a generalised Cauchy-Riemann system.

If $A$ is elliptic, then every matrix $A_{j}$ is invertible. On multiplying system (1.1) by $A_{1}^{-1}$ from the left we transform it to $C_{1} \partial_{1} u+\ldots+C_{n} \partial_{n} u=0$ where $C_{j}=A_{1}^{-1} A_{j}$, for $j=1, \ldots, n$. In particular, $C_{1}=E_{k}$ is the unit $(k \times k)$-matrix. In the sequel we will consider only those generalised Cauchy-Riemann equations (1.1), whose coefficient matrix $A_{1}$ just amounts to $E_{k}$. By the above, this assumption does not restrict generality.

Lemma 1.2. For system (1.1) with $A_{1}=E_{k}$ to be a generalised Cauchy-Riemann system it is necessary and sufficient that

$$
A_{i} A_{j}+A_{j} A_{i}=-2 \delta_{i j} E_{k}
$$

for all $i, j=2, \ldots, n$. 
Proof. See Theorems 1.2 and 1.3 in [Ste91]. Suppose (1.1) is a generalised CauchyRiemann system with $A_{1}=E_{k}$. Given any $i, j=1, \ldots, n$ with $i \neq j$, consider the function

$$
u(x)=2 x^{i} x^{j} v-\left(\left(x^{i}\right)^{2} A_{i}^{-1} A_{j}+\left(x^{j}\right)^{2} A_{j}^{-1} A_{i}\right) v,
$$

where $v$ is an arbitrary vector of $\mathbb{C}^{k}$. Obviously,

$$
\begin{aligned}
A u & =A_{i} \partial_{i} u+A_{j} \partial_{j} u \\
& =A_{i}\left(2 x^{j}-2 x^{i} A_{i}^{-1} A_{j}\right) v+A_{j}\left(2 x^{i}-2 x^{j} A_{j}^{-1} A_{i}\right) v \\
& =0,
\end{aligned}
$$

i.e., $u$ satisfies (1.1). Furthermore, $\Delta u=-2\left(A_{i}^{-1} A_{j}+A_{j}^{-1} A_{i}\right) v$, as is easy to check. Since (1.1) are generalised Cauchy-Riemann equations, we conclude that $\Delta u=0$, and so

$$
A_{i}^{-1} A_{j}+A_{j}^{-1} A_{i}=0,
$$

for $v$ can be chosen arbitrarily. Putting $i=1$ we obtain $A_{j}^{-1}=-A_{j}$ and $A_{j}^{2}=-E_{k}$ for all $j=2, \ldots, n$. On substituting these equalities into (1.3) we derive readily (1.2).

Conversely, if equalities (1.2) are fulfilled, then on setting

$$
B=E_{k} \partial_{1}-\sum_{j=2}^{n} A_{j} \partial_{j}
$$

one verifies easily that $B A=E_{k} \Delta$. Hence, $A$ is a generalised Cauchy-Riemann system, as desired.

The matrices $A_{2}, \ldots, A_{n}$ of system (1.1) with $A_{1}=E_{k}$ are immediately specified as representations of generators of the Clifford algebra $\mathcal{C}_{n-1}$ over the field $\mathbb{C}$ in the algebra of all linear mappings of $\mathbb{C}^{k}$, for they fulfil the relations (1.2). The representation of $\mathcal{C}_{n-1}$ in $\mathcal{L}\left(\mathbb{C}^{k}\right)$ is said to be reducible, if there is an invariant subspace of $\mathbb{C}^{k}$, i.e., every vector of this subspace is mapped to a vector of the same subspace by every linear transformation of the representation. Otherwise the representation is called irreducible. A representation is called completely reducible if it is irreducible or it splits up into several irreducible representations. The number $k$ is usually referred to as the representation degree. When representing the algebra $\mathcal{C}_{n-1}$, we have to distinguish two cases, whether $n$ is odd or even. For $n=2 m-1$, every representation of the Clifford algebra $\mathcal{C}_{n-1}$ is completely reducible. In this case $\mathcal{C}_{n-1}$ has precisely one irreducible representation and is isomorphic to the complete algebra $\mathcal{L}\left(\mathbb{C}^{2^{m-1}}\right)$. Every representation is actually one-to-one and its degree is a multiple of $2^{m-1}$. For $n=2 m$, each representation of the Clifford algebra $\mathcal{C}_{n-1}$ is completely reducible, too. There are two irreducible representations of degree $2^{m-1}$, and $\mathcal{C}_{n-1}$ is isomorphic to the direct sum of two algebras $\mathcal{L}\left(\mathbb{C}^{2^{m-1}}\right)$. A representation is one-to-one if and only if both irreducible representations are contained in it at least once. The degree of any representation is a multiple of $2^{m-1}$.

So we have specified all possible representation of $\mathcal{C}_{n-1}$ and the way to their identification. Hence we now know the shape of all generalised Cauchy-Riemann equations explicitly, see [Ste91].

Another characteristic property of generalised Cauchy-Riemann equations is the so-called rotational invariance. The classical Cauchy-Riemann system in the plane 
is invariant with respect to rotations of the coordinate system in the sense that if $O$ is an orthogonal $(2 \times 2)$-matrix with real entries then $u(O x)$ is holomorphic for any holomorphic function $u$. In [SW68] the property of rotational invariance is taken as definition of generalised Cauchy-Riemann equations. In the case of quadratic coefficient matrices the class introduced in [SW68] just amounts to the class considered in this section. Namely, if $O$ is an orthogonal $(n \times n)$-matrix of real numbers then $Q u(O x)$ satisfies (1.1) for each solution $u$ of this system, where $Q$ is a certain nonsingular matrix depending on $O$. For a proof, we refer the reader to $[$ Ste91, 2.4].

From the rotational invariance of generalised Cauchy-Riemann equations it follows that one rewrite them, by rotating the coordinate system $x$ if necessary, in an equivalent form $A u=0$ with $A$ satisfying $A^{*} A=-E_{k} \Delta$, where $A^{*}$ is the formal adjoint operator for $A$, see [Ste91, p. 176]. In this case, the coefficient matrices $A_{1}, \ldots, A_{n}$ are unitary. A direct argument can be obtained from the theory of group representations, cf. [Boe55].

\section{The Hilbert boundary value problem}

Let $\mathcal{X}$ be a bounded domain with smooth boundary in $\mathbb{R}^{n}$. Given a function $u_{0}$ at $\partial \mathcal{X}$ with values in $\mathbb{C}^{k / 2}$, we look for a solution $u$ to (1.1) in $\mathcal{X}$ which moreover satisfies

$$
B u=u_{0} \text { on } \partial \mathcal{X},
$$

where $B$ is a $(k / 2 \times k)$-matrix of continuous functions on $\partial \mathcal{X}$ whose rank is maximal, i.e., $k / 2$. Problem (1.1), (2.1) is usually referred to as the Hilbert boundary value problem.

Remark 2.1. Since each generalised Cauchy-Riemann operator $A$ has a fundamental solution $\Phi$ of convolution type, the inhomogeneous system $A u=f$ in $\mathcal{X}$ is reduced to the homogeneous one by substituting $u+\Phi\left(\chi_{\mathcal{X}} f\right)$ for $u$, where $\chi_{\mathcal{X}}$ is the characteristic function of $\mathcal{X}$.

The study of the Hilbert boundary value problem for general elliptic systems of first order partial differential equations in a bounded domain $\mathcal{X} \subset \mathbb{R}^{n}$ goes back at least as far as [Ava82]. The problem was reduced to a system of singular integral equations the boundary of $\mathcal{X}$ and both a condition for the Fredholm property and an index theorem were given. For generalised Cauchy-Riemann systems this result was refined in [Ste91, Ste93a, Ste93b], including explicit verification of the Shapiro-Lopatinskij condition. In [MMT11] the Hilbert boundary value problem is studied for generalised Maxwell equations. These equations have more complicated structure than (1.1).

For the classical Cauchy-Riemann equations we get $k=1$, and so $k / 2$ fails to be a whole number. To dispense with the assumption on the evenness of $k$ one can rewrite equations (1.1) in the obvious way over the field of real numbers. Then condition (2.1) takes the form $\Re\left(B^{\prime} u\right)=u_{0}$ on $\partial \mathcal{X}$, where $B^{\prime}$ is a nonsingular $(k \times k)$-matrix of continuous functions on $\partial \mathcal{X}$ and $u_{0}$ a function on the boundary with values in $\mathbb{R}^{k}$.

The rows of the matrix $B(x)$ are linearly independent at each point $x \in \partial \mathcal{X}$. On applying the Gram-Schmidt orthogonalisation one can orthonormalise them in $\mathbb{C}^{k}$. The properties of continuity and smoothness of the matrix elements do not change. 
Hence, we can assume without restriction of generality that the rows of $B(x)$ form pointwise an orthonormal system.

The task is now to find those conditions on the boundary coefficients and geometry of the domain $\mathcal{X}$ under which the Hilbert boundary value problem has the Fredholm property. When considered in the Sobolev spaces, a boundary value problem for an elliptic system is Fredholm if and only if it satisfies the ShapiroLopatinskij condition, see for instance [Vol65]. We quote the main result of [Ste91] which gives an explicit equivalent reformulation of the Shapiro-Lopatinskij condition for the Hilbert problem.

Theorem 2.2. Assume that the boundary of $\mathcal{X}$ is of class $C^{s}$, with $s \geq 1$, and the entries of $B$ of class $C^{s}(\partial \mathcal{X})$. Given any $u_{0} \in H^{s-1 / 2}\left(\partial \mathcal{X}, \mathbb{C}^{k / 2}\right)$, one looks for a solution $u \in H^{s}\left(\mathcal{X}, \mathbb{C}^{k}\right)$ to (1.1), (2.1). Then, the problem is Fredholm if and only if

$$
\operatorname{det}\left(B(\sigma(A)(\tau))^{*}(\sigma(A)(\nu)) B^{*}-\imath E_{k / 2}\right) \neq 0
$$

for all $x \in \partial \mathcal{X}$, where $\nu$ is the unit outward normal vector of the boundary at $x$, $\tau$ runs through all unit tangent vectors at $x$, and we identify vectors of $\mathbb{R}^{n}$ and its dual space.

Proof. See Theorem 3.1 in [Ste91].

In [Ste93a] Theorem 2.2 is used to establish several existence and nonexistence theorems of Fredholm boundary values problems for generalised Cauchy-Riemann equations. They contain all previously known results for special systems. If the space dimension is odd, i.e., $n=2 m-1$ with $m \geq 1$, and the number of irreducible components of the representation $A_{2}, \ldots, A_{n}$ of the Clifford algebra $\mathcal{C}_{n-1}$ is odd, i.e., $k=2^{m-1} \ell$ with $\ell$ being odd, then there does not exist any Fredholm boundary value problem of Hilbert type for the system (1.1). In the Euclidean space $\mathbb{R}^{4}$ there are no Fredholm boundary value problems of Hilbert type for those generalised Cauchy-Riemann equations which contain only one of the two possible irreducible representations. In particular, this is the case for the Fueter equations, see Section 3 . If $n$ is odd and the number of irreducible components is even or $n$ is even and both irreducible representations appear in the same number, then there exist Fredholm boundary value problems of Hilbert type for generalised Cauchy-Riemann equations in $\mathbb{R}^{n}$.

The paper [Ste93b] studies in detail the adjoint boundary value problem to apply the general theory of elliptic boundary value problems to the Hilbert problem in the case, where the Shapiro-Lopatinskij condition is fulfilled, see [Vol65] and elsewhere. This is precisely the border line over which elliptic theory no longer works, and so the boundary Fourier method in the Hilbert boundary value problem is well motivated.

\section{A Hilbert problem for the Fueter EQUations}

A natural counterpart of the classical Cauchy-Riemann system for functions of one quaternionic variable is

$$
\begin{aligned}
& \partial_{1} u^{1}-\partial_{2} u^{2}-\partial_{3} u^{3}-\partial_{4} u^{4}=0 \\
& \partial_{2} u^{1}+\partial_{1} u^{2}-\partial_{4} u^{3}+\partial_{3} u^{4}=0 \\
& \partial_{3} u^{1}+\partial_{4} u^{2}+\partial_{1} u^{3}-\partial_{2} u^{4}=0 \\
& \partial_{4} u^{1}-\partial_{3} u^{2}+\partial_{2} u^{3}+\partial_{1} u^{4}=0
\end{aligned}
$$


see [Fue36]. In this case we have $A_{1}=E_{4}$ while the corresponding matrices $A_{2}, A_{3}$ and $A_{4}$ give rise to a representation of the Clifford algebra $\mathcal{C}_{3}$ over $\mathbb{C}$ in the algebra $\mathcal{L}\left(\mathbb{C}^{4}\right)$. Since the coefficients are real, one can restrict the study to functions $u$ with values in $\mathbb{R}^{4}$.

By the above, there is no Fredholm boundary value problem of Hilbert type for equations (3.1), see also [Vin85]. We explain the character of irregularity in terms of an example.

Let $\mathcal{X}$ be a bounded domain in $\mathbb{R}^{4}$ with smooth boundary and $u_{0}^{1}$, $u_{0}^{3}$ given realvalued functions on $\partial \mathcal{X}$. We look for a function $u$ in $\mathcal{X}$ with values in $\mathbb{R}^{4}$ satisfying (3.1) and

$$
\begin{aligned}
& u^{1}=u_{0}^{1} \\
& u^{3}=u_{0}^{3}
\end{aligned}
$$

on $\partial \mathcal{X}$, which corresponds to the choice $B=\left(\begin{array}{llll}1 & 0 & 0 & 0 \\ 0 & 0 & 1 & 0\end{array}\right)$.

Introduce the standard complex structure in $\mathbb{R}^{4}$ by setting $U^{j}=u^{j}+\imath u^{2+j}$ and $z^{j}=x^{j}+\imath x^{2+j}$ for $j=1,2$. Then boundary value problem (3.1), (3.2) takes the form

$$
\begin{aligned}
& \partial_{\bar{z}^{1}} U^{1}-\partial_{z^{2}} U^{2}=0 \\
& \partial_{\bar{z}^{2}} U^{1}+\partial_{z^{1}} U^{2}=0
\end{aligned}
$$

in $\mathcal{X}$ and $U^{1}=U_{0}$ on the boundary, where $U_{0}=u_{0}^{1}+\imath u_{0}^{3}$. As usual, we define the complex derivatives by

for $j=1,2$.

$$
\begin{aligned}
\partial_{z^{j}} & =\frac{1}{2}\left(\partial_{j}-\imath \partial_{2+j}\right), \\
\partial_{\bar{z}^{j}} & =\frac{1}{2}\left(\partial_{j}+\imath \partial_{2+j}\right),
\end{aligned}
$$

Since each solution $u$ to (3.1) in $\mathcal{X}$ has harmonic components, we conclude that the component $U^{1}$ of any solution $U=\left(U^{1}, U^{2}\right)$ in $\mathcal{X}$ is uniquely defined by its restriction to the boundary. To wit, we get $U^{1}=P\left(U_{0}\right)$ in $\mathcal{X}$, where $P\left(U_{0}\right)$ is the Poisson integral of $U_{0}$. Hence, the Hilbert boundary value problem reduces to the inhomogeneous system

$$
\begin{aligned}
& \partial_{z^{1}} U^{2}=-\partial_{\bar{z}^{2}} U^{1}, \\
& \partial_{z^{2}} U^{2}=\partial_{\bar{z}^{1}} U^{1}
\end{aligned}
$$

for the second component $U_{2}$ in $\mathcal{X}$. If the domain $\mathcal{X}$ is pseudoconvex in $\mathbb{R}^{4} \equiv \mathbb{C}^{2}$, then for the solvability of (3.3) it is necessary and sufficient that the right hand side would satisfy

$$
\partial_{z^{1}} \partial_{\bar{z}^{1}} U^{1}=-\partial_{z^{2}} \partial_{\bar{z}^{2}} U^{1}
$$

which just amounts to saying that $U^{1}$ is harmonic in $\mathcal{X}$. Obviously, the solution $U^{2}$ of (3.3) is not unique and is defined up to an antiholomorphic function of $z=\left(z^{1}, z^{2}\right)$ in $\mathcal{X}$.

The main analytic difficulty in currying out this construction is that system (3.3) does not bear elliptic regularity up to the boundary of $\mathcal{X}$. More precisely, the inhomogeneous Cauchy-Riemann equations in a strongly pseudoconvex domain of $\mathbb{C}^{n}$ are subelliptic unless $n=1$. Thus, given any $u_{0}^{1}, u_{0}^{3} \in H^{1 / 2}(\partial \mathcal{X})$ with real values, we solve a Dirichlet problem to find a complex-valued harmonic function $U^{1} \in H^{1}(\mathcal{X})$ satisfying $U^{1}=U_{0}$ on $\partial \mathcal{X}$. Hence, the right-hand side of (3.3) will belong to $L^{2}\left(\mathcal{X}, \mathbb{C}^{2}\right)$ and on applying the theory of the $\bar{\partial}$-Neumann problem we get 
a solution $U^{2} \in H^{1 / 2}(\mathcal{X})$ to $(3.3)$. The regularity of $U^{2}$ can not be improved within this approach.

\section{Green Formula}

We consider a generalised Cauchy-Riemann operator $A(\partial)=A_{1} \partial_{1}+\ldots+A_{n} \partial_{n}$ satisfying $A^{*} A=-E_{k} \Delta$, where $\Delta$ is the Laplace operator in $\mathbb{R}^{n}$. Let $e(x)$ be the standard fundamental solution of convolution type for $\Delta$, i.e., $e(x)=(2 \pi)^{-1} \log |x|$, if $n=2$, and

$$
e(x)=\frac{1}{\sigma_{n}} \frac{1}{2-n} \frac{1}{|x|^{n-2}},
$$

if $n \geq 3$, where $\sigma_{n}$ is the surface area of the unit sphere in $\mathbb{R}^{n}$.

Lemma 4.1. The matrix $\Phi=-A^{*} e$ is a (two-sided) fundamental solution of convolution type of the operator $A$, i.e., $\Phi A=A \Phi=I$ on compactly supported distributions in $\mathbb{R}^{n}$ with values in $\mathbb{C}^{k}$.

Proof. This follows by a trivial verification.

The boundary Fourier method is based on an integral identity specifying the complementary part of $B u$ in the Cauchy data of $u$ on the boundary of $\mathcal{X}$ relative to the generalised Cauchy-Riemann operator $A(\partial)=A_{1} \partial_{1}+\ldots+A_{n} \partial_{n}$. By the above, there is no loss of generality in assuming that the rows of the matrix $B(x)$ are pointwise orthonormal. Then there is a $(k / 2 \times k)$-matrix $C$ of smooth functions on $\partial \mathcal{X}$, such that the matrix

$$
T(x)=\left(\begin{array}{l}
B(x) \\
C(x)
\end{array}\right)
$$

is unitary for all $x \in \partial \mathcal{X}$.

Lemma 4.2. There are unique matrices $B^{\text {adj }}$ and $C^{\text {adj }}$ of continuous functions on $\partial \mathcal{X}$ with the property that

$$
\int_{\partial \mathcal{X}}\left(\left(B u, C^{\mathrm{adj}} g\right)_{x}-\left(C u, B^{\mathrm{adj}} g\right)_{x}\right) d s=\int_{\mathcal{X}}\left((A u, g)_{x}-\left(u, A^{*} g\right)_{x}\right) d x
$$

for all $u \in H^{1}\left(\mathcal{X}, \mathbb{C}^{k}\right)$ and $g \in H^{1}\left(\mathcal{X}, \mathbb{C}^{k}\right)$, where $d s$ is the surface measure on the boundary.

Proof. Since $T(x)$ is a unitary matrix for all $x \in \partial \mathcal{X}$, we get $T^{*} T=E_{k}$, which is equivalent to $B^{*} B+C^{*} C=E_{k}$.

Given any $u \in H^{1}\left(\mathcal{X}, \mathbb{C}^{k}\right)$ and $g \in H^{1}\left(\mathcal{X}, \mathbb{C}^{k}\right)$, the Green formula of [Tar95, 9.2.2] shows that

$$
\int_{\partial \mathcal{X}}(\sigma u, g)_{x} d s=\int_{\mathcal{X}}\left((A u, g)_{x}-\left(u, A^{*} g\right)_{x}\right) d x
$$

where $\sigma(x):=\sigma(A)(-\imath \nu(x))$ for $x \in \partial \mathcal{X}$. On substituting $u=\left(B^{*} B+C^{*} C\right) u$ into this formula yields (4.1) with

$$
\begin{aligned}
C^{\text {adj }} & =B \sigma^{*}, \\
B^{\text {adj }} & =-C \sigma^{*},
\end{aligned}
$$

as desired. 
From (4.2) it follows immediately that the ranks of both $C^{\text {adj }}$ and $B^{\text {adj }}$ are equal to $k / 2$.

Formula (4.1) is said to be a Green formula related to the boundary value problem $\{A, B\}$. The formula is not uniquely determined by the pair $\{A, B\}$, for the complementary part $C$ of $B$ in the Cauchy data $\{B, C\}$ can be chosen in many ways. On choosing $C$ we fix a duality on the manifold with boundary $\overline{\mathcal{X}}$ associated with $\{A, B\}$. The problem

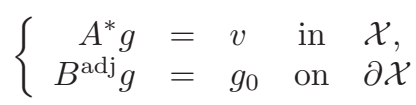

is called adjoint to $\{A, B\}$ with respect to the Green formula. Clearly, (4.3) is of Hilbert type, too.

From the Green formula it follows that for the solvability of problem (1.1), (2.1) it is necessary that

$$
\int_{\partial \mathcal{X}}\left(u_{0}, C^{\mathrm{adj}} g\right)_{x} d s=0
$$

for all $g \in H^{1}\left(\mathcal{X}, \mathbb{C}^{k}\right)$ which satisfy the homogeneous problem corresponding to (4.3), i.e., $A^{*} g=0$ in $\mathcal{X}$ and $B^{\text {adj }} g=0$ on $\partial \mathcal{X}$. The moment conditions (4.4) specify a closed space of boundary data $u_{0}$ which contains the range of the Hilbert boundary value problem. However, this space need not have finite codimension, for the space of solutions of the homogeneous adjoint problem may be infinite dimensional, cf. Section 3.

\section{HARdy SPACES}

For a smooth function $f$ in $\mathcal{X}$, we set $f_{\varepsilon}(y):=f(y-\varepsilon \nu(y))$, where $\nu(y)$ is the unit outward normal to $\partial \mathcal{X}$ at $y$. So $f_{\varepsilon}$ is a family of smooth functions on $\partial \mathcal{X}$ parametrised by a small parameter $\varepsilon>0$. We say that $f$ admits a distribution boundary value on $\partial \mathcal{X}$, if

$$
\lim _{\varepsilon \rightarrow 0+} \int_{\partial \mathcal{X}} f_{\varepsilon} g d s=:\left\langle f_{0}, g\right\rangle
$$

exists for all $g \in C_{\text {comp }}^{\infty}(\partial \mathcal{X})$. In this case the limit defines a distribution $f_{0}$ on the boundary and the convergence is not only in the weak sense but also in the strong topology on $\mathcal{D}^{\prime}(\partial \mathcal{X})$.

The local structure of harmonic functions admitting distribution boundary values is well known.

Theorem 5.1. For a harmonic function $f$ in $\mathcal{X}$, the following properties are equivalent:

1) $f$ admits a distribution boundary value on $\partial \mathcal{X}$.

2) $f$ is in the Sobolev space $H^{-s}(\mathcal{X})$, for some integer $s$.

3) There exist an integer $N$ and $C>0$, such that $|f(x)| \leq C /(\operatorname{dist}(x, \partial \mathcal{X}))^{N}$ for all $x \in \mathcal{X}$.

4) For any $x_{0} \in \partial \mathcal{X}$ there are a neighbourhood $U$ in $\mathbb{R}^{n}$ and a function $h$ harmonic in $U \cap \mathcal{X}$ and continuous in $U \cap \overline{\mathcal{X}}$, such that $f=\langle c, \partial\rangle^{N} h$ in $U \cap \mathcal{X}$, where $c \in \mathbb{R}^{n}$ is a constant vector and $N$ an integer.

Proof. See [Str84, Theorem 1.1]. The proof actually shows that, for a harmonic function $f$ which admits a distribution boundary value on $\partial \mathcal{X}$, this boundary value 
is the trace of $f$ on $\partial \mathcal{X}$ in the sense of Sobolev spaces, i.e., $f_{0} \in H^{-s-1 / 2}(\partial \mathcal{X})$ provided $f \in H^{-s}(\mathcal{X})$.

From Theorem 5.1 it follows that if $f$ is a harmonic function in $\mathcal{X}$ which admits a distribution boundary value on $\partial \mathcal{X}$ then

$$
\sum_{|\alpha| \leq m} b_{\alpha}(x) \partial^{\alpha} f
$$

also admits a distribution boundary value on $\partial \mathcal{X}$ whenever the coefficients $b_{\alpha}$ are $C^{\infty}$ in the closure of $\mathcal{X}$. It is worth pointing out that this function need not be harmonic.

Just as in the case of more familiar harmonic Hardy spaces, the Poisson integral mediates between boundary values and the corresponding harmonic functions. Given a distribution $f_{0}$ on $\partial \mathcal{X}$, we write $P\left(f_{0}\right)$ for the Poisson integral of $f_{0}$. It is defined by $P\left(f_{0}\right)(x)=\left\langle P(x, \cdot), f_{0}\right\rangle$ for $x \in \mathcal{X}$, where $P(x, y)$ is the Poisson kernel for $\mathcal{X}$, i.e., the normal derivative of the Green function $G(x, y)$ at $y \in \partial \mathcal{X}$. For each integer $s$, the Poisson integral induces an isomorphism of $H^{-s-1 / 2}(\partial \mathcal{X})$ onto the subspace of $H^{-s}(\mathcal{X})$ consisting of harmonic functions in $\mathcal{X}$. Its inverse is the map assigning to each harmonic function $f \in H^{-s}(\mathcal{X})$ its boundary value, see Corollary 1.7 in [Str84].

Yet another designation for functions in $\mathcal{X}$, which are polynomially bounded in $1 / \operatorname{dist}(x, \partial \mathcal{X})$, is functions of finite order of growth near the boundary, cf. Chapter 9 in [Tar95] and elsewhere. By the above, a harmonic function of finite order of growth near the boundary in $\mathcal{X}$ is uniquely determined by its distribution boundary value on $\partial \mathcal{X}$. This allows one to identify harmonic functions of finite order of growth in $\mathcal{X}$ with their boundary values on $\partial \mathcal{X}$. In this way we obtain many interesting Banach spaces of harmonic functions in the domain $\mathcal{X}$. The most popular of them is perhaps the Hardy space $\mathcal{H}^{2}(\mathcal{X})$. This space is defined to consist of all harmonic functions in $\mathcal{X}$ of finite order of growth near the boundary, whose distribution boundary values on $\partial \mathcal{X}$ belong to $L^{2}(\partial \mathcal{X})$. When endowed with $L^{2}(\partial \mathcal{X})$-norm, $\mathcal{H}^{2}(\mathcal{X})$ is a Hilbert space.

Let now $u$ be a smooth function in $\mathcal{X}$ with values in $\mathbb{C}^{k}$ satisfying a generalised Cauchy-Riemann system $A u=0$ in $\mathcal{X}$. If there exist an integer $N$ and $C>0$, such that $|u(x)| \leq C /(\operatorname{dist}(x, \partial \mathcal{X}))^{N}$ for all $x \in \mathcal{X}$, then the same is true for the components of $u$. By Theorem 5.1, each component admits a distribution boundary value on $\partial \mathcal{X}$. Hence, $u$ admits a boundary value on $\partial \mathcal{X}$ which is a continuous linear functional on $C_{\text {comp }}^{\infty}\left(\partial \mathcal{X}, \mathbb{C}^{k}\right)$. Moreover, both $B u$ and $C u$ admit boundary values on $\partial \mathcal{X}$ which are distributions with values in $\mathbb{C}^{k}$. This is precisely the sense in which we interpret them in the following formula analogous to the Cauchy integral formula.

Lemma 5.2. For each solution $u$ to equations (1.1) in $\mathcal{X}$ of finite order of growth near $\partial \mathcal{X}$, it follows that

$-\int_{\partial \mathcal{X}}\left(\left(B u, C^{\operatorname{adj}} \Phi(x-\cdot)^{*}\right)_{y}-\left(C u, B^{\operatorname{adj}} \Phi(x-\cdot)^{*}\right)_{y}\right) d s= \begin{cases}u(x), & \text { if } x \in \mathcal{X}, \\ 0, & \text { if } x \in \mathbb{R}^{n} \backslash \overline{\mathcal{X}}\end{cases}$

Note that $(\Phi(x-y))^{*}=(A e)(x-y)$ for all $x$ and $y$ away from the diagonal of $\mathbb{R}^{n}$, as is easy to check. 
Proof. See Theorem 9.4.1 of [Tar95].

This reasoning, when looked at from a more general point of view, leads to new investigations of Fredholm boundary value problems in Hardy spaces, see [Tar95, 11.2.2].

\section{Part 2. Boundary Fourier method}

\section{Operator-TheOREtic FOUNDATIONS}

The operator-theoretic foundations of the method of Fischer-Riesz equations are elaborated in [Tar95, 11.1]. It goes back at least as far as [PF50]. Here we adapt this method for studying the Hilbert boundary value problem for generalised Cauchy-Riemann equations.

Any solution of generalised Cauchy-Riemann equations in $\mathcal{X}$ is a $k$-column of harmonic functions in this domain. Therefore, the $k$-fold product of the Hardy space

$$
\mathcal{H}^{2}(\mathcal{X})^{k}=\underbrace{\mathcal{H}^{2}(\mathcal{X}) \times \ldots \times \mathcal{H}^{2}(\mathcal{X})}_{k \text { times }}
$$

fits well to constitute the domain of problem (1.1), (2.1). More precisely, denote by $H_{1}$ the vector space of all $u \in \mathcal{H}^{2}(\mathcal{X})^{k}$ satisfying $A u=0$ in $\mathcal{X}$. When endowed with the $L^{2}\left(\partial \mathcal{X}, \mathbb{C}^{k}\right)$-norm, this space is complete, i.e., a Hilbert space. Besides, set $H_{2}=L^{2}\left(\partial \mathcal{X}, \mathbb{C}^{k / 2}\right)$ and

$$
H=H_{2} \times L^{2}\left(\partial \mathcal{X}, \mathbb{C}^{k / 2}\right) .
$$

Consider the mapping $M: H_{1} \rightarrow H$ given by $M u=(B u, C u)$, which corresponds to the Cauchy problem for solutions of $A u=0$ in $\mathcal{X}$ with Cauchy data $B u=u_{0}$ and $C u=u_{1}$ on $\partial \mathcal{X}$. By the above, $M$ is continuous. In Section 7 we will prove that $M$ has closed range.

Denote by $M^{*}: H \rightarrow H_{1}$ the operator that is adjoint to $M: H_{1} \rightarrow H$ in the sense of Hilbert spaces.

Lemma 6.1. The null-space $\operatorname{ker} M^{*}$ of the operator $M^{*}$ is separable in the topology induced from $H$.

Proof. This is true by the school fact that any subspace of a separable metric space is separable.

Let $\mathcal{S}_{A^{*}}(\overline{\mathcal{X}})$ stand for the space of all solutions to the formal adjoint system $A^{*} g=0$ on neighbourhoods of $\overline{\mathcal{X}}$. Since $A^{*}$ is elliptic, these are real analytic functions with values in $\mathbb{C}^{k}$.

Lemma 6.2. Assume that $g \in \mathcal{S}_{A^{*}}(\overline{\mathcal{X}})$. Then the couple $\left(C^{\text {adj }} g,-B^{\text {adj }} g\right)$ belongs to $\operatorname{ker} M^{*}$.

Proof. One has to show that $\left(M u,\left(C^{\text {adj }} g,-B^{\text {adj }} g\right)\right)_{H}=0$ for all $u \in H_{1}$. By the Green formula, we get

$$
\begin{aligned}
\left(M u,\left(C^{\text {adj }} g,-B^{\text {adj }} g\right)\right)_{H} & =\int_{\partial \mathcal{X}}\left(\left(B u, C^{\text {adj }} g\right)_{x}-\left(C u, B^{\text {adj }} g\right)_{x}\right) d s \\
& =0,
\end{aligned}
$$

as desired. 
The subspace of ker $M^{*}$ consisting of all elements of the form $\left(C^{\text {adj }} g,-B^{\text {adj }} g\right)$, where $g \in \mathcal{S}_{A^{*}}(\overline{\mathcal{X}})$, is separable. Hence, there are many ways to choose a sequence $\left\{g_{i}\right\}_{i=1,2, \ldots}$ in $\mathcal{S}_{A^{*}}(\overline{\mathcal{X}})$, such that the system $\left\{\left(C^{\text {adj }} g_{i},-B^{\text {adj }} g_{i}\right)\right\}$ is complete in this subspace.

In Example 6.5 we will show some explicit sequences $\left\{g_{i}\right\}$ with this property. For the moment we fix one of such sequences.

Lemma 6.3. As defined above, the system $\left\{\left(C^{\text {adj }} g_{i},-B^{\text {adj }} g_{i}\right)\right\}_{i=1,2, \ldots}$ is complete in $\operatorname{ker} M^{*}$.

Proof. Let $\mathcal{F}$ be a continuous linear functional on ker $M^{*}$ vanishing on each element of the system $\left\{\left(C^{\text {adj }} g_{i},-B^{\text {adj }} g_{i}\right)\right\}$. Since ker $M^{*}$ is a closed subspace of $H$, the Riesz representation theorem implies the existence of an element $\left(u_{0}, u_{1}\right) \in \operatorname{ker} M^{*}$, such that the action of $\mathcal{F}$ on $\operatorname{ker} M^{*}$ consists in scalar multiplication with the element $\left(u_{0}, u_{1}\right)$. In particular,

$$
\begin{aligned}
\mathcal{F}\left(C^{\text {adj }} g_{i},-B^{\text {adj }} g_{i}\right) & =\int_{\partial \mathcal{X}}\left(\left(C^{\text {adj }} g_{i}, u_{0}\right)_{x}-\left(B^{\text {adj }} g_{i}, u_{1}\right)_{x}\right) d s \\
& =0
\end{aligned}
$$

for all $i=1,2, \ldots$. Since the system $\left\{\left(C^{\text {adj }} g_{i},-B^{\text {adj }} g_{i}\right)\right\}_{i=1,2, \ldots}$ is dense in the subspace of $\operatorname{ker} M^{*}$ consisting of all elements of the form $\left(C^{\text {adj }} g,-B^{\text {adj }} g\right)$, where $g \in \mathcal{S}_{A^{*}}(\overline{\mathcal{X}})$, we get

$$
\int_{\partial \mathcal{X}}\left(\left(u_{0}, C^{\mathrm{adj}} g\right)_{x}-\left(u_{1}, B^{\mathrm{adj}} g\right)_{x}\right) d s=0
$$

for all $g \in \mathcal{S}_{A^{*}}(\overline{\mathcal{X}})$. We now use Theorem 7.1 which says that there exists a function $u \in \mathcal{H}^{2}(\mathcal{X})^{k}$ such that $A u=0$ in $\mathcal{X}$ and $B u=u_{0}, C u=u_{1}$ at the boundary of $\mathcal{X}$. In other words, $\left(u_{0}, u_{1}\right)=M u$. Hence it follows that $\mathcal{F}(h)=(h, M u)_{H}=0$ for all $h \in \operatorname{ker} M^{*}$. Thus, $\mathcal{F} \equiv 0$ and the standard application of the Hahn-Banach theorem completes the proof.

Write $P$ for the orthogonal projection of $H$ onto the first factor $H_{2}$. The composition $P M=B$ acting from $H_{1}$ to $H_{2}$ just amounts to the operator of boundary value problem $(1.1),(2.1)$ in the updated setting. More precisely, given any $u_{0} \in L^{2}\left(\partial \mathcal{X}, \mathbb{C}^{k / 2}\right)$, find $u \in \mathcal{H}^{2}(\mathcal{X})^{k}$ satisfying $A u=0$ in $\mathcal{X}$ and $B u=u_{0}$ weakly on the boundary of $\mathcal{X}$. The following lemma expresses the most important property of the system $\left\{g_{i}\right\}$.

Lemma 6.4. The system $\left\{B^{\mathrm{adj}} g_{i}\right\}_{i=1,2, \ldots}$ is complete in $L^{2}\left(\partial \mathcal{X}, \mathbb{C}^{k / 2}\right)$ if and only if $P M$ is injective.

Proof. By the Hahn-Banach theorem, $\left\{B^{\text {adj }} g_{i}\right\}$ is complete in $L^{2}\left(\partial \mathcal{X}, \mathbb{C}^{k / 2}\right)$ if and only if any continuous linear functional $\mathcal{F}$ on $L^{2}\left(\partial \mathcal{X}, \mathbb{C}^{k / 2}\right)$ vanishing on each element of the system, is zero. Pick such a functional $\mathcal{F}$. By the Riesz representation theorem there is a function $u_{1} \in L^{2}\left(\partial \mathcal{X}, \mathbb{C}^{k / 2}\right)$ such that $\mathcal{F}(h)=\left(h, u_{1}\right)$ for all $h \in L^{2}\left(\partial \mathcal{X}, \mathbb{C}^{k / 2}\right)$. So we get

$$
\begin{aligned}
\left(\left(0, u_{1}\right),\left(C^{\mathrm{adj}} g_{i},-B^{\mathrm{adj}} g_{i}\right)\right)_{H} & =-\overline{\left(B^{\mathrm{adj}} g_{i}, u_{1}\right)_{L^{2}\left(\partial \mathcal{X}, \mathbb{C}^{k / 2}\right)}} \\
& =-\overline{\mathcal{F}\left(B^{\mathrm{adj}} g_{i}\right)} \\
& =0
\end{aligned}
$$


for all $i=1,2, \ldots$. Applying Lemma 6.3 we deduce that the element $\left(0, u_{1}\right)$ belongs to the orthogonal complement of the subspace ker $M^{*}$ in $H$. Since the operator $M$ has closed range, the orthogonal complement of ker $M^{*}$ coincides with the range of $M$. Hence, there is a function $u \in \mathcal{H}^{2}(\mathcal{X})^{k}$ satisfying $A u=0$ in $\mathcal{X}$ and $B u=0$, $C u=u_{1}$ on $\partial \mathcal{X}$. If the operator $P M$ is injective, then $u=0$ whence $u_{1}=0$ and $\mathcal{F}=0$. Conversely, if the functional $\mathcal{F}$ is different from zero, then $u_{1}$ is not zero and so the operator $P M$ fails to be injective, which is precisely the desired conclusion.

After removing the elements which are linear combinations of the previous ones from the system $\left\{B^{\text {adj }} g_{i}\right\}_{i=1,2, \ldots}$, we get a sequence $\left\{g_{i_{n}}\right\}$ in $\mathcal{S}_{A^{*}}(\overline{\mathcal{X}})$, such that the system $\left\{B^{\text {adj }} g_{i_{n}}\right\}$ is linearly independent. Applying then the Gram-Schmidt orthogonalisation to the system $\left\{B^{\text {adj }} g_{i_{n}}\right\}$ in $L^{2}\left(\partial \mathcal{X}, \mathbb{C}^{k / 2}\right)$, we obtain a new system $\left\{e_{n}\right\}_{n=1,2, \ldots}$ in $\mathcal{S}_{A^{*}}(\overline{\mathcal{X}})$, such that $\left\{B^{\text {adj }} e_{n}\right\}$ is an orthonormal system in the space $L^{2}\left(\partial \mathcal{X}, \mathbb{C}^{k / 2}\right)$. Moreover, $\left\{B^{\text {adj }} e_{n}\right\}$ is an orthonormal basis in $L^{2}\left(\partial \mathcal{X}, \mathbb{C}^{k / 2}\right)$, provided that $P M$ is injective. Note that the elements $e_{n}$ of the new system have explicit expressions through the elements $\left\{g_{i_{1}}, \ldots, g_{i_{n}}\right\}$ of the old system in the form of Gram's determinants.

Example 6.5. Since $\mathcal{X}$ is a bounded domain with smooth boundary, its complement has only finitely many connected components. Let $\left\{x_{i}\right\}$ be a finite set of points in $\mathbb{R}^{n} \backslash \overline{\mathcal{X}}$, such that each connected component of $\mathbb{R}^{n} \backslash \overline{\mathcal{X}}$ contains at least one point $x_{i}$. Then the columns of the matrix $\partial^{\alpha} \Phi\left(x_{i}-\cdot\right)^{*}$ belong to $\mathcal{S}_{A^{*}}(\overline{\mathcal{X}})$ and the system $\left\{B^{\text {adj }} \partial^{\alpha} \Phi\left(x_{i}-\cdot\right)^{*}\right\}$ is complete in the subspace of $L^{2}\left(\mathcal{X}, \mathbb{C}^{k / 2}\right)$ formed by elements of the type $B^{\text {adj }} g$ with $g \in \mathcal{S}_{A^{*}}(\overline{\mathcal{X}})$.

The proof of this fact actually repeats the reasoning of Example 11.4.14 in [Tar95]. Apparently the system of Example 6.5 is most convenient for numerical simulations.

\section{The Cauchy problem}

For $u \in \mathcal{H}^{2}(\mathcal{X})^{k}$, the Green formula (4.1) displays the Cauchy data of $u$ on the boundary of $\mathcal{X}$ with respect to the operator $A$. These are weak limit values of $B u$ and $C u$ at $\partial \mathcal{X}$. Hence we formulate the Cauchy problem as follows: Given any $u_{0} \in L^{2}\left(\partial \mathcal{X}, \mathbb{C}^{k / 2}\right)$ and $u_{1} \in L^{2}\left(\partial \mathcal{X}, \mathbb{C}^{k / 2}\right)$, find a function $u \in \mathcal{H}^{2}(\mathcal{X})^{k}$ satisfying $A u=0$ in $\mathcal{X}$ and

on $\partial \mathcal{X}$.

$$
\left\{\begin{array}{l}
B u=u_{0} \\
C u=u_{1}
\end{array}\right.
$$

The Cauchy problem for solutions of systems with injective symbol and data on the whole boundary was intensively studied in the 1960s. This study was motivated to a certain extent by the paper [Cal63]. The study of the Cauchy problem in Hardy spaces is motivated by the problem of analytic continuation, cf. Chapter 11 in [Tar95].

Theorem 7.1. Let $u_{0}, u_{1} \in L^{2}\left(\partial \mathcal{X}, \mathbb{C}^{k / 2}\right)$ be given functions. In order that there might exist a solution $u \in \mathcal{H}^{2}(\mathcal{X})^{k}$ to $A u=0$ in $\mathcal{X}$ subject to (7.1), it is necessary and sufficient that

$$
\int_{\partial \mathcal{X}}\left(\left(u_{0}, C^{\text {adj }} g\right)_{x}-\left(u_{1}, B^{\text {adj }} g\right)_{x}\right) d s=0
$$


for all $g \in \mathcal{S}_{A^{*}}(\overline{\mathcal{X}})$.

Proof. Necessity. If $u \in \mathcal{H}^{2}(\mathcal{X})^{k}$ is a solution of the Cauchy problem with data $u_{0}$, $u_{1}$, then by the Green formula

$$
\begin{aligned}
\int_{\partial \mathcal{X}}\left(\left(u_{0}, C^{\mathrm{adj}} g\right)_{x}-\left(u_{1}, B^{\mathrm{adj}} g\right)_{x}\right) d s & =\int_{\partial \mathcal{X}}\left(\left(B u, C^{\mathrm{adj}} g\right)_{x}-\left(C u, B^{\mathrm{adj}} g\right)_{x}\right) d s \\
& =0
\end{aligned}
$$

for all $g \in \mathcal{S}_{A^{*}}(\overline{\mathcal{X}})$, as required.

Sufficiency. We introduce a function $U$ in $\mathcal{X} \backslash \partial \mathcal{X}$ with values in $\mathbb{C}^{k}$ by the Green-type integral

$$
U(x)=-\int_{\partial \mathcal{X}}\left(\left(u_{0}, C^{\operatorname{adj}} \Phi(x-\cdot)^{*}\right)_{y}-\left(u_{1}, B^{\text {adj }} \Phi(x-\cdot)^{*}\right)_{y}\right) d s,
$$

where $x \in \mathcal{X} \backslash \partial \mathcal{X}$. An easy calculation using (4.2) shows that

$$
\left(u_{0}, C^{\text {adj }} \Phi(x-\cdot)^{*}\right)_{y}-\left(u_{1}, B^{\text {adj }} \Phi(x-\cdot)^{*}\right)_{y}=\Phi(x-\cdot)\left(\sigma u_{b}\right)
$$

on $\partial \mathcal{X}$, where

$$
u_{b}=B^{*} u_{0}+C^{*} u_{1}
$$

Since

$$
\left(\begin{array}{l}
B \\
C
\end{array}\right)\left(B^{*}, C^{*}\right)=\left(\begin{array}{ll}
B B^{*} & B C^{*} \\
C B^{*} & C C^{*}
\end{array}\right)=E_{k},
$$

we get $B u_{b}=u_{0}$ and $C u_{b}=u_{1}$, and so $u_{b}$ is of class $L^{2}\left(\partial \mathcal{X}, \mathbb{C}^{k}\right)$ if and only if both $u_{0}$ and $u_{1}$ belong to $L^{2}\left(\partial \mathcal{X}, \mathbb{C}^{k / 2}\right)$. Thus, formula (7.3) reduces to

$$
U=-\Phi *\left([\partial \mathcal{X}] \sigma u_{b}\right)
$$

in $\mathcal{X} \backslash \partial \mathcal{X}$.

For each fixed $x \in \mathcal{X} \backslash \overline{\mathcal{X}}$, the columns of the matrix $\Phi(x-\cdot)^{*}$ belong to $\mathcal{S}_{A^{*}}(\overline{\mathcal{X}})$. Hence, (7.2) implies that $U$ vanishes in the complement of $\overline{\mathcal{X}}$.

Set $u=U\lceil\mathcal{X}$. We next prove that $u$ is the desired solution of the Cauchy problem. This is equivalent to saying that $u \in \mathcal{H}^{2}(\mathcal{X})^{k}$ and $A u=0$ in $\mathcal{X}, u \uparrow_{\partial \mathcal{X}}=u_{b}$ at $\partial \mathcal{X}$.

From the structure of the fundamental matrix $\Phi$ it follows immediately that $u$ belongs to $\mathcal{H}^{2}(\mathcal{X})^{k}$ and satisfies $A u=0$ in $\mathcal{X}$. Since $u_{b} \in L^{2}\left(\partial \mathcal{X}, \mathbb{C}^{k}\right)$, the jump of the double layer potential $\Phi\left([\partial \mathcal{X}] \sigma u_{b}\right)$ under crossing the surface $\partial \mathcal{X}$ from $\mathcal{X} \backslash \overline{\mathcal{X}}$ to $\mathcal{X}$ just amounts to $u_{b}$. This is true even for all distributions $u_{b}$ on $\partial \mathcal{X}$ taking their values in $\mathbb{C}^{k}$, see Theorem 10.1.5 in [Tar95]. For the square integrable densities $u_{b}$ the jump is understood in an appropriate sense including the $L^{2}\left(\partial \mathcal{X}, \mathbb{C}^{k}\right)$-norm. Summarising we conclude that $u \uparrow_{\partial \mathcal{X}}=u_{b}$, for $U$ vanishes in $\mathcal{X} \backslash \overline{\mathcal{X}}$. This completes the proof.

\section{The Fischer-Riesz equations}

Let $\left\{g_{i}\right\}_{i=1,2, \ldots}$ be an arbitrary sequence in $\mathcal{S}_{A^{*}}(\overline{\mathcal{X}})$ with the property that the system $\left\{\left(C^{\text {adj }} g_{i},-B^{\text {adj }} g_{i}\right)\right\}$ is complete in ker $M^{*}$. Applying the Gram-Schmidt orthogonalisation to the system $\left\{B^{\text {adj }} g_{i}\right\}$ in $L^{2}\left(\partial \mathcal{X}, \mathbb{C}^{k / 2}\right)$, we obtain a new system $\left\{e_{n}\right\}_{n=1,2, \ldots}$ in $\mathcal{S}_{A^{*}}(\overline{\mathcal{X}})$, such that the system $\left\{B^{\text {adj }} e_{n}\right\}$ is orthonormal in the space $L^{2}\left(\partial \mathcal{X}, \mathbb{C}^{k / 2}\right)$. 
Given any $u_{1} \in L^{2}\left(\partial \mathcal{X}, \mathbb{C}^{k / 2}\right)$, we denote by $k_{n}\left(u_{1}\right)$ the Fourier coefficients of $u_{1}$ with respect to the system $\left\{B^{\text {adj }} e_{n}\right\}$, i.e.,

$$
k_{n}\left(u_{1}\right)=\int_{\partial \mathcal{X}}\left(u_{1}, B^{\mathrm{adj}} e_{n}\right)_{y} d s
$$

for $n=1,2, \ldots$.

Lemma 8.1. If $u \in \mathcal{H}^{2}(\partial \mathcal{X})^{k}$ satisfies $A u=0$ in $\mathcal{X}$, then

$$
k_{n}(C u)=\int_{\partial \mathcal{X}}\left(B u, C^{\operatorname{adj}} e_{n}\right)_{y} d s
$$

where $n=1,2, \ldots$.

Proof. Using Lemma 6.2 we obtain

$$
\begin{aligned}
k_{n}(C u) & =\int_{\partial \mathcal{X}}\left(C u, B^{\mathrm{adj}} e_{n}\right)_{y} d s+\left(M u,\left(C^{\mathrm{adj}} e_{n},-B^{\mathrm{adj}} e_{n}\right)\right)_{H} \\
& =\int_{\partial \mathcal{X}}\left(B u, C^{\mathrm{adj}} e_{n}\right)_{y} d s
\end{aligned}
$$

as desired.

Thus, in order to find the Fourier coefficients of the data $C u$ on the boundary with respect to the system $\left\{B^{\text {adj }} e_{n}\right\}$ in $L^{2}\left(\partial \mathcal{X}, \mathbb{C}^{k / 2}\right)$, it suffices to know only the data $B u$ of problem (1.1), (2.1).

Theorem 8.2. Let $u_{0} \in L^{2}\left(\partial \mathcal{X}, \mathbb{C}^{k / 2}\right)$. In order that there be a $u \in \mathcal{H}^{2}(\mathcal{X})^{k}$ such that $A u=0$ in $\mathcal{X}$ and $B u=u_{0}$ on $\partial \mathcal{X}$, it is necessary and sufficient that

1) $\sum_{n=1}^{\infty}\left|c_{n}\right|^{2}<\infty$, where $c_{n}=\int_{\partial \mathcal{X}}\left(u_{0}, C^{\mathrm{adj}} e_{n}\right)_{y} d s$, and ary.

2) $\int_{\partial \mathcal{X}}\left(u_{0}, C^{\text {adj }} g\right)_{y} d s=0$ for all $g \in \mathcal{S}_{A^{*}}(\overline{\mathcal{X}})$ satisfying $B^{\text {adj }} g=0$ on the bound-

Proof. Necessity. Suppose there is a function $u \in \mathcal{H}^{2}(\mathcal{X})^{k}$ satisfying $A u=0$ in $\mathcal{X}$ and $B u=u_{0}$ at $\partial \mathcal{X}$. Then $c_{n}=k_{n}(C u)$ for all $n=1,2, \ldots$, which is due to Lemma 8.1. Applying the Bessel inequality yields

$$
\sum_{n=1}^{\infty}\left|c_{n}\right|^{2}=\sum_{n=1}^{\infty}\left|k_{n}(C u)\right|^{2} \leq \int_{\partial \mathcal{X}}|C u|^{2} d s<\infty,
$$

and 1) is proved. On the other hand, 2) follows immediately from the Green formula, as desired.

Sufficiency. We now assume that 1) and 2) are satisfied. Condition 1) implies, by the Fischer-Riesz theorem, that there exists a function $u_{1} \in L^{2}\left(\partial \mathcal{X}, \mathbb{C}^{k / 2}\right)$, such that $\left\{c_{n}\right\}_{n=1,2, \ldots}$ are the Fourier coefficients of $u_{1}$ with respect to the orthonormal system $\left\{B^{\text {adj }} e_{n}\right\}$ in $L^{2}\left(\partial \mathcal{X}, \mathbb{C}^{k / 2}\right)$. In other words, we get $c_{n}=k_{n}\left(u_{1}\right)$ for all $n=1,2, \ldots$. On substituting formulas for $c_{n}$ from 1 ) into these equalities we arrive at the orthogonality relations

$$
\int_{\partial \mathcal{X}}\left(\left(u_{0}, C^{\mathrm{adj}} e_{n}\right)_{y}-\left(u_{1}, B^{\mathrm{adj}} e_{n}\right)_{y}\right) d s=0
$$

for $n=1,2, \ldots$, cf. (7.2). 
Our next goal is to prove that the element $\left(u_{0}, u_{1}\right) \in H$ is actually orthogonal to all elements of the system $\left\{\left(C^{\text {adj }} g_{i},-B^{\text {adj }} g_{i}\right)\right\}_{i=1,2, \ldots}$ in $H$, this latter being complete in ker $M^{*}$. To do this, let us recall how the system $\left\{e_{n}\right\}$ has been obtained from the system $\left\{g_{i}\right\}$.

Even if the system $\left\{\left(C^{\text {adj }} g_{i},-B^{\text {adj }} g_{i}\right)\right\}$ is linearly independent in $H$, the system $\left\{B^{\text {adj }} g_{i}\right\}$ may have elements which are linear combinations of the previous ones in the space $L^{2}\left(\partial \mathcal{X}, \mathbb{C}^{k / 2}\right)$. Such elements should be eliminated from the system before applying the Gram-Schmidt orthogonalisation.

For example, suppose that, for some $i$, the equality

$$
B^{\mathrm{adj}} g_{i}=\sum_{j=1}^{i-1} c_{i, j} B^{\mathrm{adj}} g_{j}
$$

is fulfilled with suitable complex numbers $c_{i, j}$. Consider the function

$$
g_{i}^{\prime}=g_{i}-\sum_{j=1}^{i-1} c_{i, j} g_{j}
$$

which belongs to $\mathcal{S}_{A^{*}}(\overline{\mathcal{X}})$. Obviously, $\left(C^{\text {adj }} g_{i}^{\prime},-B^{\text {adj }} g_{i}^{\prime}\right)$ lies in ker $M^{*}$ and satisfies $B^{\text {adj }} g_{i}^{\prime}=0$. It follows that

$$
g_{i}=\sum_{j=1}^{i-1} c_{i, j} g_{j}+g_{i}^{\prime} .
$$

All the other elements $\left(C^{\text {adj }} g_{i},-B^{\text {adj }} g_{i}\right.$, except for the eliminated ones, are expressed, by the contents of Gram-Schmidt orthogonalisation, as linear combinations of the elements $\left\{\left(C^{\text {adj }} e_{n},-B^{\text {adj }} e_{n}\right)\right\}_{n=1, \ldots, i}$. Thus, any element of the system $\left\{\left(C^{\text {adj }} g_{i},-B^{\text {adj }} g_{i}\right)\right\}$ has a unique expression through the elements of the system $\left\{\left(C^{\operatorname{adj}} e_{n},-B^{\operatorname{adj}} e_{n}\right)\right\}_{n=1,2, \ldots}$ in the form

$$
g_{i}=\sum_{n=1}^{i} c_{i, n} e_{n}+g_{i}^{\prime},
$$

where $g_{i}^{\prime} \in \mathcal{S}_{A^{*}}(\overline{\mathcal{X}})$ satisfies $B^{\text {adj }} g_{i}^{\prime}=0$ on the boundary $\partial \mathcal{X}$.

From equalities (8.1) and (8.2) and condition 2$)$ of the theorem it follows immediately that

$$
\begin{aligned}
& \left(\left(u_{0}, u_{1}\right),\left(C^{\text {adj }} g_{i},-B^{\text {adj }} g_{i}\right)\right)_{H} \\
& \quad=\sum_{n=1}^{i} c_{i, n}\left(\left(u_{0}, u_{1}\right),\left(C^{\text {adj }} e_{n},-B^{\text {adj }} e_{n}\right)\right)_{H}+\left(\left(u_{0}, u_{1}\right),\left(C^{\text {adj }} g_{i}^{\prime},-B^{\text {adj }} g_{i}^{\prime}\right)\right)_{H} \\
& \quad=0
\end{aligned}
$$

for all $i=1,2, \ldots$. Since the system $\left\{\left(C^{\text {adj }} g_{i},-B^{\text {adj }} g_{i}\right)\right\}_{i=1,2, \ldots}$ is complete in ker $M^{*}$, the element $\left(u_{0}, u_{1}\right)$ belongs to the orthogonal complement of this subspace in $H$. Using the lemma of operator kernel annihilator, we deduce that there exists a function $u \in H_{1}$ satisfying $M u=\left(u_{0}, u_{1}\right)$. In particular, $u \in \mathcal{H}^{2}(\mathcal{X})^{k}$ satisfies $A u=0$ in $\mathcal{X}$ and $B u=u_{0}$ on $\partial \mathcal{X}$, i.e., $u$ is the desired solution of boundary value problem (1.1), (2.1). 
The convergence of the series in 1) guarantees the stability of boundary value problem (1.1), (2.1). Under this condition, the range of the mapping $P M$ is described in terms of continuous linear functionals on the space $H$, cf. 2), which is impossible in the general case.

Corollary 8.3. Under the hypotheses of Theorem 8.2, if moreover the homogeneous adjoint boundary value problem (4.3) has no smooth solutions in $\overline{\mathcal{X}}$ different from zero, then for problem (1.1), (2.1) to possess a solution $u \in \mathcal{H}^{2}(\mathcal{X})^{k}$ it is necessary and sufficient that

$$
\sum_{n=1}^{\infty}\left|c_{n}\right|^{2}<\infty
$$

Proof. This follows immediately from Theorem 8.2 since condition 2) is automatically fulfilled.

\section{Regularisation of SOlutions}

Note that the proof of Theorem 8.2 works without the assumption that the operator $P M$ in $H$ is injective. Our next objective will be to construct an approximate solution to boundary value problem $(1.1),(2.1)$. To this end it is natural to assume that the corresponding homogeneous boundary value problem has only zero solution in the space $\mathcal{H}^{2}(\mathcal{X})^{k}$, i.e., the mapping $P M$ is injective. In this case the orthonormal system $\left\{B^{\text {adj }} e_{n}\right\}$ is actually complete in the space $L^{2}\left(\partial \mathcal{X}, \mathbb{C}^{k / 2}\right)$. The orthonormal bases in $L^{2}\left(\partial \mathcal{X}, \mathbb{C}^{k / 2}\right)$ of this form are said to be special, cf. [Tar95, 11.3].

For $x \in \mathcal{X} \backslash \partial \mathcal{X}$, we denote by $k_{n}\left(B^{\operatorname{adj}} \Phi(x-\cdot)^{*}\right)$ the $k$-row whose entries are the Fourier coefficients of the columns of the $((k / 2) \times k)$-matrix $B^{\text {adj }} \Phi(x-\cdot)^{*}$ with respect to the orthonormal basis $\left\{B^{\text {adj }} e_{n}\right\}_{n=1,2, \ldots}$ in $L^{2}\left(\partial \mathcal{X}, \mathbb{C}^{k / 2}\right)$. More precisely, we set

for $n=1,2, \ldots$.

$$
k_{n}\left(B^{\mathrm{adj}} \Phi(x-\cdot)^{*}\right)=\int_{\partial \mathcal{X}}\left(B^{\mathrm{adj}} \Phi(x-\cdot)^{*}, B^{\mathrm{adj}} e_{n}\right)_{y} d s
$$

Lemma 9.1. For $n=1,2, \ldots$, the coefficients $k_{n}\left(B^{\operatorname{adj}} \Phi(x-\cdot)^{*}\right)$ are analytic functions in $\mathcal{X} \backslash \partial \mathcal{X}$ with values in $\left(\mathbb{C}^{k}\right)^{*}$.

Proof. The assertion is obvious, for the fundamental solution $\Phi(x-y)$ is analytic away from the diagonal of $\mathcal{X} \times \mathcal{X}$.

Consider the following (Schwartz) kernels $R_{N}$ defined for $x \in \mathcal{X} \backslash \partial \mathcal{X}$ and $y$ in a neighbourhood of $\overline{\mathcal{X}}$ :

$$
R_{N}(x, y)=\Phi(x-y)-\sum_{n=1}^{N} k_{n}\left(B^{\operatorname{adj}} \Phi(x-\cdot)^{*}\right)^{*} e_{n}(y)^{*},
$$

where $N=1,2, \ldots$

Lemma 9.2. As defined above, the kernels $R_{N}$ are analytic in $x \in \mathcal{X} \backslash \partial \mathcal{X}$ and $y$ in a neighbourhood of $\overline{\mathcal{X}}$ except for the diagonal $\{x=y\}$, and $A^{*}(y, D) R_{N}(\cdot-y)^{*}=0$ on this set.

Proof. This follows immediately from Lemma 9.1 and the fact that $e_{n} \in \mathcal{S}_{A^{*}}(\overline{\mathcal{X}})$, as desired. 
The sequence $\left\{R_{N}\right\}$ provides a very special approximation of the fundamental solution $\Phi$.

Lemma 9.3. The sequence $\left\{B^{\operatorname{adj}} R_{N}(x, \cdot)^{*}\right\}_{N=1,2, \ldots}$ converges to zero in the norm of $L^{2}\left(\partial \mathcal{X}, \mathbb{C}^{(k / 2) \times k}\right)$ uniformly in $x$ on compact subsets of $\mathcal{X} \backslash \partial \mathcal{X}$.

Proof. In fact, we get

$$
\begin{aligned}
B^{\text {adj }} R_{N}(x, \cdot)^{*} & =B^{\text {adj }} \Phi(x-\cdot)^{*}-\sum_{n=1}^{N} B^{\operatorname{adj}} e_{n} k_{n}\left(B^{\operatorname{adj}} \Phi(x-\cdot)^{*}\right) \\
& =\sum_{n=N+1}^{\infty} B^{\operatorname{adj}} e_{n} k_{n}\left(B^{\operatorname{adj}} \Phi(x-\cdot)^{*}\right)
\end{aligned}
$$

for each fixed $x \in \mathcal{X} \backslash \partial \mathcal{X}$. The right-hand side of this equality is a remainder of the Fourier series of the element $B^{\text {adj }} R_{N}(x, \cdot)^{*}$ with respect to the orthonormal basis $\left\{B^{\text {adj }} e_{n}\right\}$ in $L^{2}\left(\partial \mathcal{X}, \mathbb{C}^{k / 2}\right)$. Hence, it tends to zero in the $L^{2}\left(\partial \mathcal{X}, \mathbb{C}^{(k / 2) \times k}\right)$-norm, as $N \rightarrow \infty$. This proves the first part of the lemma. The second part follows from a general remark on Fourier series, for the mapping of $\mathcal{X} \backslash \partial \mathcal{X}$ to $L^{2}\left(\partial \mathcal{X}, \mathbb{C}^{(k / 2) \times k}\right)$ given by

$$
x \mapsto B^{\operatorname{adj}} \Phi(x-\cdot)^{*}
$$

is continuous.

The convergence of the approximations allows one to reconstruct solutions $u$ of the class $\mathcal{H}^{2}\left(\mathcal{X}, \mathbb{C}^{k}\right)$ to $A u=0$ in $\mathcal{X}$ through their data $B u$.

Theorem 9.4. Every function $u \in \mathcal{H}^{2}(\mathcal{X})^{k}$ satisfying $A u=0$ in $\mathcal{X}$ can be represented by the integral formula

$$
u(x)=\lim _{N \rightarrow \infty}\left(-\int_{\partial \mathcal{X}}\left(B u, C^{\text {adj }} R_{N}(x, \cdot)^{*}\right)_{y} d s\right)
$$

for all $x \in \mathcal{X}$.

Proof. Fix a point $x \in \mathcal{X}$. Since $R_{N}(x, \cdot)^{*}$ and $\Phi(x-\cdot)^{*}$ differ by a $k$-row of smooth solutions of the system $A^{*} g=0$ in a neighbourhood of $\overline{\mathcal{X}}$, one can write by the Green formula

$$
u(x)=-\int_{\partial \mathcal{X}}\left(\left(B u, C^{\text {adj }} R_{N}(x, \cdot)^{*}\right)_{y}-\left(C u, B^{\operatorname{adj}} R_{N}(x, \cdot)^{*}\right)_{y}\right) d s
$$

for any $N=1,2, \ldots$. From $u \in \mathcal{H}^{2}(\mathcal{X})^{k}$ we deduce that $C u \in L^{2}\left(\partial \mathcal{X}, \mathbb{C}^{k / 2}\right)$. Hence it follows by Lemma 9.3 that

$$
\lim _{N \rightarrow \infty} \int_{\partial \mathcal{X}}\left(C u, B^{\mathrm{adj}} R_{N}(x, \cdot)^{*}\right)_{y} d s=0 .
$$

Thus, letting $N \rightarrow \infty$ in (9.1) establishes the formula.

As mentioned, for many problems of mathematical physics formulas for approximate solution like that of Theorem 9.4 were earlier obtained by Kupradze and his colleagues, see [Kup67]. 


\section{Solvability of Elliptic Boundary VAlue PROBlems}

We can now return to the Sobolev space setting of boundary value problem (1.1), (2.1) which is $H_{1}=H^{1}\left(\mathcal{X}, \mathbb{C}^{k}\right)$. Given any $u \in H^{1}\left(\mathcal{X}, \mathbb{C}^{k}\right)$, both $A u$ and $B u$ are well defined in $L^{2}\left(\mathcal{X}, \mathbb{C}^{k}\right)$ and $H^{1 / 2}\left(\partial \mathcal{X}, \mathbb{C}^{k / 2}\right)$, respectively. Hence, the analysis does not require any function spaces of negative smoothness. More generally, let $s$ be a natural number. Given any $u_{0}$ in $H^{s-1 / 2}\left(\partial \mathcal{X}, \mathbb{C}^{k / 2}\right)$, we look for a $u \in H^{s}\left(\mathcal{X}, \mathbb{C}^{k}\right)$ satisfying (1.1), (2.1). Theorem 8.2 still applies to establish the existence of a weak solution $u \in \mathcal{H}^{2}(\mathcal{X})^{k}$, provided that the conditions 1) and 2) are fulfilled. To infer the existence of a Sobolev space solution, one needs a regularity theorem for weak solutions in $\mathcal{H}^{2}(\mathcal{X})^{k}$ saying that any weak solution belongs actually to the Sobolev space $H^{s}\left(\mathcal{X}, \mathbb{C}^{k}\right)$. This is the case if (1.1), (2.1) is an elliptic boundary value problem, i.e., the pair $\{A, B\}$ satisfies the Shapiro-Lopatinskij condition at the boundary of $\mathcal{X}$.

Corollary 10.1. Suppose a regularity theorem holds for boundary value problem (1.1), (2.1). Let $u_{0} \in H^{s-1 / 2}\left(\partial \mathcal{X}, \mathbb{C}^{k / 2}\right)$, where $s=1,2, \ldots$ Then, in order that there be $a u \in H^{s}\left(\mathcal{X}, \mathbb{C}^{k}\right)$ satisfying $A u=0$ in $\mathcal{X}$ and $B u=u_{0}$ on $\partial \mathcal{X}$ it is necessary and sufficient that

$$
\begin{aligned}
& \text { 1) } \sum_{n=1}^{\infty}\left|c_{n}\right|^{2}<\infty \text {, where } c_{n}=\int_{\partial \mathcal{X}}\left(u_{0}, C^{\text {adj }} e_{n}\right)_{y} d s \text {, and } \\
& \text { 2) } \int_{\partial \mathcal{X}}\left(u_{0}, C^{\text {adj }} g\right)_{y} d s=0 \text { for all } g \in \mathcal{S}_{A^{*}}(\overline{\mathcal{X}}) \text { satisfying } B^{\text {adj }} g=0 \text { at the bound- }
\end{aligned}
$$

Proof. It is sufficient to prove the sufficiency of conditions 1) and 2). If the conditions 1) and 2) are satisfied, then there exists a function $u \in \mathcal{H}^{2}(\mathcal{X})^{k}$, such that $A u=0$ in $\mathcal{X}$ and $B u=u_{0}$ on $\partial \mathcal{X}$. For solutions of $A u=0$ in $\mathcal{X}$ the condition $u \in \mathcal{H}^{2}(\mathcal{X})^{k}$ just amounts to saying that $u \in H^{1 / 2}\left(\mathcal{X}, \mathbb{C}^{k}\right)$. Since $A u \in H^{s-1}\left(\mathcal{X}, \mathbb{C}^{k}\right)$ and $B u \in H^{s-1 / 2}\left(\partial \mathcal{X}, \mathbb{C}^{k / 2}\right)$, the regularity theorem implies that $u \in H^{s}\left(\mathcal{X}, \mathbb{C}^{k}\right)$, as desired.

If $\{A, B\}$ is elliptic then so is the problem $\left\{A^{*}, B^{\text {adj }}\right\}$ adjoint to $\{A, B\}$ with respect to the Green formula. By the Fredholm property, the space of all $g \in$ $\mathcal{S}_{A^{*}}(\overline{\mathcal{X}})$ satisfying $B^{\mathrm{adj}} g=0$ on $\partial \mathcal{X}$, is finite dimensional. Moreover, the condition 2) alone is sufficient for the existence of a solution $u \in H^{s}\left(\mathcal{X}, \mathbb{C}^{k}\right)$ to problem (1.1), (2.1). Hence it follows that for elliptic boundary value problems the condition 1) is automatically fulfilled.

Thus, the regularity problem for weak solutions of (1.1), (2.1) is still of primary character in the study of boundary value problems. On the other hand, our approach demonstrates rather strikingly that Theorem 9.4 is of great importance for numerical simulation. 


\section{REFERENCES}

[AT13] Alsaedy, A., and Tarkhanov, N., The method of Fischer-Riesz equations for elliptic boundary value problems, J. of Complex Analysis 1 (2013), Issue 1, 1-11.

[Ava82] Avantaggiati, A., Internal and external first order boundary value problems on $C^{1}$ domains, Pubblicazioni Serie III, 215, IAC "Mauro Picone", Rome, 1982, 19 pp.

[Bal02] Balabaev, V. E., The ellipticity problem for systems of first-order partial differential equations, Differential Equations 38 (2002), No. 3, 434-436.

[BDT89] Baum, P., Douglas, R., and Taylor, M., Cycles and relative cycles in $K$-homology, J. Diff. Geometry 30 (1989), 761-804.

[Boe55] Boerner, H., Darstellungen von Gruppen, Springer-Verlag, Berlin, 1955.

[BW92] Booss, B., and Wojciechowsky, K., Boundary Value Problems for Dirac Operators, Birkhäuser, Boston, 1992.

[BDS82] Bracks, F., Delange, R., and Sommen, F., Clifford Analysis, Pitman, Boston et al., 1982.

[Cal63] Calderón, A. P., Boundary value problems for elliptic equations, In: "Outlines Joint Symposium PDE" (Novosibisrk, 1963), Acad. Sci. USSR, Siberian Branch, Moscow, 1963, 303-304.

[Fed74] Fedosov, B. V., Analytic formulas for the index of elliptic operators, Trans. Moscow Math. Soc. 30 (1974), 159-240.

[Fre56] Fréchet, M., A paraanalytic function in $n$ variables, J. Reine Angew. Math. 193 (1956), 122-141.

[Fue36] Fueter, R., Theorie der regülären Funktionen einer Quaternionenvariablen, In: Oslo Comptes Rendus de Congress International des Mathematiciens, 1936, 75-91.

[Gak77] Gakhov, F. D., Boundary Value Problems, Nauka, Moscow, 1977.

[GM90] Gilbert, J., and Murray, M., Clifford Algebras and Dirac Operators in Harmonic Analysis, Cambridge Univ. Press, Cambridge, 1990.

[Gon93] Gong, G., Relative $K$-cycles and elliptic boundary conditions, Bull. Amer. Math. Soc. 28 (1993), 104-108.

[Kup67] Kupradze, V. D., Approximate solution of problems of mathematical physics, Uspekhi Mat. Nauk 22 (1967), No. 2, 59-107.

[MMT11] Makhmudov, K., Makhmudov, O., and Tarkhanov, N., Equations of Maxwell type, J. Math. Anal. Appl. 378 (2011), Issue 1, 64-75.

[MT31] Moisil, G. C., and Theodorescu, N., Fonction holomorphic dans l'espace, Bul. Soc. St. Cluj 6 (1931), 177-194.

[Mus46] Muskhelishvili, N. I., Singular Integral Equations, P. Noordhoff, Groningen, 1946.

[OT08] Osetrova, T., and Tarkhanov, N., Algebraic analysis of differential operators, J. of Siberian Federal University, Mathematics and Physics 1 (2008), No. 4, 391-398.

[PF50] Picone, M., and Fichera, G., Neue funktional-analytische Grundlagen für die Existenzprobleme und Lösungsmethoden von Systemen linearer partieller Differentialgleichungen, Monatsh. Math. 54 (1950), 188-209.

[SV95] Shapiro, M., and Vasilevski, N., Quaternionic hyperholomorphic functions, singular integral operators and boundary value problems, I, Complex Variables 27 (1995), 17-44; II, Complex Variables 27 (1995), 67-96.

[Shn72] Shnirelman, A. I., The degree of a quasiruled mapping and a nonlinear Hilbert problem, Math. USSR Sb. 18 (1972), 373-396.

[SW68] Stein, E., and Weiss, G., Generalization of Cauchy-Riemann equations and representations of the rotation group, Amer. J. Math. 90 (1968), 163-196.

[Ste91] Stern, I., Boundary value problems for generalized Cauchy-Riemann systems in the space, In: "Boundary value and initial value problems in complex analysis: Studies in complex analysis and its applications to partial differential equations, I" (Halle, 1988), Pitman Res. Notes Math. Ser., Vol. 256, Longman Sci. Tech., Harlow, 1991.

[Ste93a] Stern, I., On the existence of Fredholm boundary value problems for generalized Cauchy-Riemann systems, Complex Variables 21 (1993), 19-38.

[Ste93b] Stern, I., Direct methods for generalized Cauchy-Riemann systems in the space, Complex Variables 23 (1993), 73-100.

[Str84] Straube, E. J., Harmonic and analytic functions admitting a distribution boundary value, Ann. Scuola Norm. Super. Pisa 11 (1984), No. 4, 559-591. 
[Tar95] Tarkhanov, N., The Cauchy Problem for Solutions of Elliptic Equations, Akademie Verlag, Berlin, 1995.

[Vek62] Vekua, I. N., Generalized Analytic Functions, Pergamon Press, Oxford, 1962.

[Vin85] Vinogradov, V. S., A boundary value problem for pseudosymmetric systems, Differential Equations 21 (1985), No. 1, 161-163.

[Vol65] Volevich, L. R., On the solvability of boundary value problems for general elliptic systems, Mat. Sb. 68 (110) (1965), No. 3, 373-416.

[Weg92] Wegert, E., Nonlinear Boundary Value Problems for Holomorphic Functions and Singular Integral Equations, Akademie Verlag, Berlin, 1992.

Department of Mathematics, College of Science, Alnahrain University, Baghdad, IRAQ

E-mail address: ammar.alsaedy@ymail.com

Currently in Institute for Mathematics, University of Potsdam, Karl-LiebknechtStr. 24/25, 14476 Potsdam (Golm), Germany

E-mail address: alsaedy@math.uni-potsdam.de

Institute of Mathematics, University of Potsdam, Karl-Liebknecht-Str. 24/25, 14476 Potsdam (Golm), Germany

E-mail address: tarkhanov@math.uni-potsdam.de 